\title{
Safety and efficacy of Pazopanib in advanced soft tissue sarcoma: PALETTE (EORTC 62072) subgroup analyses
}

\author{
Axel Le Cesne ${ }^{1 *}$, Sebastian Bauer ${ }^{2}$, George D. Demetri ${ }^{3}$, Guangyang Han ${ }^{4}$, Luca Dezzanii ${ }^{4}$, Qasim Ahmad ${ }^{4}$, \\ Jean-Yves Blay ${ }^{5}$, lan Judson ${ }^{6}$, Patrick Schöffski ${ }^{7}$, Massimo Aglietta ${ }^{8}$, Peter Hohenberger ${ }^{9}$ and Hans Gelderblom ${ }^{10}$
}

\begin{abstract}
Background: PALETTE is a phase 3 trial that demonstrated single-agent activity of pazopanib in advanced soft tissue sarcomas (aSTS). We performed retrospective subgroup analyses to explore potential relationships between patient characteristics, prior lines of therapy, dose intensity, and dose modifications on safety and efficacy of pazopanib in aSTS.

Methods: PALETTE compared pazopanib with placebo in patients with aSTS (age $\geq 18$ years) whose disease had progressed during or following prior chemotherapy. In these subgroup analyses, median progression-free survival (mPFS) among patients receiving pazopanib was the efficacy outcome of interest. Adverse events (AEs) were also compared within subgroups. All analyses were descriptive and exploratory.

Results: A total of 246 patients received pazopanib in the PALETTE study. The MPFS was longer in patients who had only 1 prior line versus 2+ prior lines of therapy (24.7 vs 18.9 weeks, respectively); AE rates were similar regardless of number of prior lines of therapy. The mPFS was similar in patients aged $<65$ and $\geq 65$ y $(20.0$ and 20.1 weeks, respectively). Although AEs leading to study discontinuation were higher in older patients ( $\geq 65 \mathrm{y}, 30 \%$; $<65 \mathrm{y}, 17 \%)$, rates of dose reductions, dose interruptions, and serious AEs were similar between the 2 age groups. No reduction in MPFS was noted in patients requiring dose reductions or dose interruptions to manage toxicities. Conclusions: Longer mPFS was observed in patients receiving pazopanib following only 1 line of therapy. Additionally, mPFS with pazopanib was maintained regardless of patient age or dose modifications used to manage toxicity.
\end{abstract}

Trial registration: NCT00753688, first posted September 16, 2008 (registered prospectively).

Keywords: Pazopanib, Advanced soft tissue sarcoma, Progression-free survival, PALETTE subgroup analysis

\section{Background}

Pazopanib is an oral, small-molecule tyrosine kinase inhibitor (TKI) targeting vascular endothelial growth factor (VEGF) receptors (VEGFR-1, - 2, and -3), platelet-derived growth factor (PDGF) receptors (PDGFR-

\footnotetext{
* Correspondence: Axel.LECESNE@gustaveroussy.fr

Previous Publication: A portion of this work was presented as a poster at the 2017 ESMO Annual Meeting: Le Cesne A, et al. Safety and efficacy of pazopanib (PAZ) in advanced soft tissue carcinoma (aSTS) by prior lines of therapy, age, and dose modifications: PALETTE subgroup analyses. Ann Oncol. 2017;28(suppl 5): Abstract 1501P. https://doi.org/10.1093/annonc/ mdx387.027. Accessed October 22, 2018.

'Département d'Oncologie Médicale, Gustave Roussy, 114 Rue Edouard Vaillant, 94805 Villejuif Cedex, Villejuif, France

Full list of author information is available at the end of the article
}

alpha and -beta), fibroblast growth factor receptor, and KIT [1]. The predominant role of VEGF and PDGF in tumor angiogenesis and their expression across many soft tissue sarcoma (STS) subtypes provided a strong rationale for the evaluation of pazopanib in STS. In a placebo-controlled, randomized, phase 3 trial in patients with advanced STS (aSTS; excluding liposarcomas and gastrointestinal stromal tumor [GIST]), pazopanib administration led to significantly improved progressionfree survival (PFS) compared with placebo [2]. These results led to the US regulatory approval of pazopanib for treating patients with aSTS who have previously received chemotherapy [3]. The EMA has approved pazopanib for adults with selected subtypes of aSTS

(c) The Author(s). 2019 Open Access This article is distributed under the terms of the Creative Commons Attribution 4.0 International License (http://creativecommons.org/licenses/by/4.0/), which permits unrestricted use, distribution, and reproduction in any medium, provided you give appropriate credit to the original author(s) and the source, provide a link to the Creative Commons license, and indicate if changes were made. The Creative Commons Public Domain Dedication waiver (http://creativecommons.org/publicdomain/zero/1.0/) applies to the data made available in this article, unless otherwise stated. 
following prior chemotherapy for metastatic disease or after progression within 12 months after (neo)adjuvant therapy [4]. Pazopanib was the first molecularly targeted agent approved for treating aSTS at a time when treatment options following failure of first-line chemotherapy (the most common first-line treatment) were very limited.

The incidence of STS increases with age, with approximately $50 \%$ of newly diagnosed patients being older than 65 years [5]. In addition to the high-grade and/or highstage STS presentation in elderly patients versus younger patients $[6,7]$, poor prognosis in older patients might also relate to undertreatment based on misconceptions of tolerability and safety [8]. Older patients are less likely to be treated with adjuvant chemotherapy, radiotherapy, or definitive surgery [9]. Also, comorbidities are generally more common in older rather than younger patients. Underrepresentation of elderly patients further compromises the generalizability of the clinical trial findings to clinical practice [10]. Number of prior lines of therapy also influences STS outcomes. In a retrospective study evaluating novel targeted therapies in patients with aSTS after progression from US FDA-approved therapies, patients who had received 2 or fewer prior lines of treatment had substantially improved overall survival (OS) in comparison with patients who had received 3 or more prior lines of treatment [11].

Flexibility of pazopanib dosing in patients with aSTS may be crucial for optimal treatment and tolerability in this setting. In the PALETTE trial, dose interruptions and dose reductions were allowed to manage adverse events (AEs). Pazopanib treatment was temporarily interrupted in $49 \%$ of patients, and $39 \%$ of patients received dose reductions. A definitive treatment discontinuation due to AEs related to pazopanib occurred in $14 \%$ of patients [2]. However, limited data have been published to date on pazopanib efficacy and safety in patients undergoing dose interruptions and dose reductions.

Using data from the PALETTE trial, we investigated pazopanib efficacy and safety in specific subgroups of patients with aSTS. Understanding the influence of age, prior lines of therapy, dosing intensity, and dose modifications on pazopanib outcomes could potentially alleviate safety and tolerability concerns and guide optimal usage of pazopanib in patients with aSTS.

\section{Methods}

\section{Study design}

PALETTE (EORTC 62072) was a randomized, doubleblind, placebo-controlled, phase 3 trial, conducted by the Soft Tissue and Bone Sarcoma Group of the European Organization for Research and Treatment of Cancer between October 2008 and November 2010. Patients were randomized 2:1 to receive either pazopanib $800 \mathrm{mg}$ once daily or placebo, with no subsequent cross-over. As part of the original study [2], all patients provided written informed consent and the trial was approved by all relevant review bodies. Because the subgroup analyses used existing data from the primary study, additional consent was not required. The study was conducted in accordance with the Declaration of Helsinki and Good Clinical Practice guidelines. Full details of the PALETTE study design, including inclusion and exclusion criteria, have been published previously [2].

Subgroups of interest in our current post hoc analyses were based on the following metrics: prior lines of therapy (only 1 prior line of therapy vs $2+$ prior lines of therapy), age (<65 years vs $\geq 65$ years) dose intensity (dose $<400 \mathrm{mg}, \geq 400 \mathrm{mg}$ to $<600 \mathrm{mg}$, and $\geq 600 \mathrm{mg}$ to $\leq 800 \mathrm{mg}$ ), and dose modifications (no dose reduction vs dose reductions; no dose interruption vs dose interruptions) among patients randomized to receive pazopanib in the PALETTE trial. All subgroup analyses were exploratory and descriptive in nature, with no statistical hypothesis testing.

\section{Eligibility criteria}

Key inclusion criteria included patients of age $\geq 18$ years with aSTS and disease progression within 6 months prior to receiving study drug or within 12 months of previous adjuvant treatment, $\geq 1$ regimen containing anthracycline, and $\leq 4$ lines of prior systemic therapy for metastatic disease. No more than 2 previous lines should have been combination regimens, and (neo)adjuvant/maintenance treatments were not counted toward this criterion. Key exclusion criteria included patients with adipocytic sarcoma, embryonal rhabdomyosarcoma, chondrosarcoma, osteosarcoma, and GIST. Patients with clinically abnormal cardiac function or poorly controlled hypertension were also excluded. Patients who had had a cerebrovascular accident, pulmonary embolism, untreated deep venous thrombosis, or clinically significant gastrointestinal disorders in the past 6 months were ineligible.

\section{Criteria for dose modifications}

Dose interruptions or reductions were permitted following potential drug-related toxicities including but not limited to hypertension, proteinuria, hepatotoxicity, bleeding events, thrombosis, and thrombocytopenia/ neutropenia. In cases wherein a dose reduction was necessary, 2 stepwise dose reductions were permitted: initially to $600 \mathrm{mg}$ and subsequently to $400 \mathrm{mg}$. If the toxicity did not recur or worsen, the doses could be increased stepwise back to $600 \mathrm{mg}$ and $800 \mathrm{mg}$ after monitoring for 10-14 days at each step. If a patient's treatment had been interrupted $>14$ days due to toxicity, resumption of treatment was based on patient's condition and recovery from toxicity at reduced dose. An additional table provides a detailed description of 
protocol-defined and prespecified dose modifications for potential treatment-related AEs (see Additional file 1).

\section{Study endpoints}

The primary objective of the PALETTE trial was to demonstrate superiority in PFS of pazopanib over placebo. In this subgroup analysis, the efficacy outcome of interest was median PFS (mPFS) among pazopanib recipients in the PALETTE trial. Adverse events were also compared within the subgroups.

\section{Statistical analysis}

Efficacy was evaluated in the intent-to-treat population, which included all patients who were randomized to treatment. Although the PALETTE study was stratified according to number of previous lines of systemic therapy and was powered to detect a $15 \%$ difference in PFS (pazopanib versus placebo arms) at 6 months, the study was not powered for any subgroup analyses. The safety population, which was defined as all patients who were administered their allocated treatment and had received at least 1 dose of the study drug, was used for all safety analyses.

\section{Results}

\section{Patient characteristics}

A total of 246 patients were randomized to the pazopanib arm and represented the intention-to-treat (ITT) population. At baseline, the mean age was 54 $( \pm 15)$ years, $60 \%$ of patients were female, and $25 \%$ of the patients were $\geq 65$ years of age (Table 1 ). At the time of the primary analysis, median follow-up was 14.9 months (interquartile range, 11.0-18.2) in the pazopanib group; disease progression was documented in 168 recipients and 137 patients had died.

\section{Subgroup analysis by age}

Among patients receiving pazopanib, 184 patients were $<65$ years of age and 62 patients were $\geq 65$ years of age. The mPFS was similar in the 2 age subgroups (age < 65 years, 20.0 [95\% CI, 17.9-22.0] weeks and age $\geq 65$ years, 20.1 [95\% CI, 11.7-31.6] weeks, respectively). Treatment-related AEs occurred in $93 \%$ of patients $<65$ years of age versus $85 \%$ of patients $\geq 65$ years of age (Table 2). The AEs leading to study discontinuation occurred at a higher rate in older $(\geq 65$ years) versus younger (<65 years) patients $(30 \%$ vs $17 \%$, respectively). However, rates of dose reductions, dose interruptions, and serious AEs leading to study discontinuation were similar between the 2 age groups (Table 2).

\section{Subgroup analysis by prior lines of therapy}

Among patients receiving pazopanib, 110 patients had received 1 prior line of therapy and 136 patients had
Table 1 Baseline Characteristics of Pazopanib Recipients in the PALETTE Trial (ITT Population)

\begin{tabular}{ll}
\hline & $\begin{array}{l}\text { Pazopanib Arm } \\
(n=246)\end{array}$ \\
\hline Age, y, mean (SD) & $54.0(14.9)$ \\
$<65$ y, n (\%) & $184(75)$ \\
$\geq 65$ y, n (\%) & $62(25)$ \\
Female, n (\%) & $147(60)$ \\
Weight, kg, mean (SD) & $71.5(16.9)$ \\
STS subtypes, n (\%) & \\
Leiomyosarcoma & $109(44)$ \\
Synovial sarcoma & $25(10)$ \\
Other STS histologies & $112(46)$ \\
Time since initial diagnosis, mo, median (IQR) & $26.6(14.5-46.1)$ \\
Time since last progression, mo, median (IQR) & $0.7(0.3-1.1)$ \\
Prior lines of therapy, $n$ (\%) & \\
1 & $110(45)$ \\
\hline
\end{tabular}

$S D$ standard deviation, STS soft tissue sarcoma, IQR interquartile range

received 2 or more prior lines of therapy. The mPFS was higher in patients with 1 prior line of therapy (24.7 weeks [95\% CI, 19.6-27.4]) versus patients with 2 or more prior lines of therapy (18.9 weeks [95\% CI, $11.9-$ 20.1]). Rates of AEs were similar between the 2 subgroups (Table 3).

\section{Subgroup analysis by dose intensity}

Among patients receiving pazopanib, the majority $(n=234)$ received doses between 600 and $800 \mathrm{mg}$ daily. Only 4 patients received doses between 400 and $600 \mathrm{mg}$ daily, and 7 patients received doses less than $400 \mathrm{mg}$ daily. Among patients receiving the highest doses, mPFS was 20.1 weeks (95\% CI, 17.9-21.3). Patients who received between 400 and $600 \mathrm{mg}$ pazopanib daily had mPFS of 25.3 weeks $(95 \%$ CI, 8.1-38.1), and mPFS was 5.1 weeks (95\% CI, 3.3-7.0) in patients who received less than $400 \mathrm{mg}$ pazopanib daily.

\section{Subgroup analysis by dose modifications}

Among patients receiving pazopanib, patients with 1 or more dose reductions had substantially higher mPFS than patients with no dose reductions (27.7 weeks [95\% CI, 21.1-35.7] vs 11.9 weeks [95\% CI, 8.9-19.3], respectively) (Table 4). Similarly, patients with 1 or more dose interruptions had substantially higher mPFS than patients with no dose interruptions (21.3 weeks [95\% CI, 20.1-27.7] vs 11.0 weeks [95\% CI, 8.1-19.3], respectively) (Table 4).

\section{Discussion}

In this PALETTE subgroup analysis, the magnitude of clinical benefit observed with pazopanib was similar 
Table 2 Adverse Events in Pazopanib Recipients by Age Subgroups (Safety Population)

\begin{tabular}{lll}
\hline Events, $\mathrm{n}(\%)$ & $\begin{array}{l}\text { Age }<65 \text { Years } \\
(n=180)\end{array}$ & $\begin{array}{c}\text { Age } \geq 65 \text { Years } \\
(n=60)\end{array}$ \\
\hline Any on-therapy AE & $178(99)$ & $59(98)$ \\
AEs related to study treatment & $168(93)$ & $51(85)$ \\
AEs leading to permanent discontinuation or early withdrawal & $30(17)$ & $18(30)$ \\
AEs leading to dose reduction & $60(33)$ & $17(28)$ \\
AEs leading to dose interruption/delay & $89(49)$ & $31(52)$ \\
Any SAE & $75(42)$ & $24(40)$ \\
SAEs related to study treatment & $40(22)$ & $17(28)$ \\
Fatal SAEs & $7(4)$ & $1(2)$ \\
Fatal SAEs related to study treatment & $1(<1)$ & $0(0)$ \\
\hline
\end{tabular}

$A E$ adverse event, SAE serious adverse event.

between elderly and younger patients. Increasing age was apparently unrelated to mPFS. Higher rates of AEs leading to study discontinuation in older patients are not surprising, likely due to a higher prevalence of comorbidities and reduced tolerability in elderly compared with younger patients. However, rates of dose reductions, dose interruptions, and serious AEs were similar between the two age groups. Retrospective analyses of patients receiving other therapies for aSTS have reported similar findings of a higher rate of AEs in elderly patients compared with their younger counterparts $[12,13]$. Because the AE profile of each agent may vary, the individual safety profile of each agent should inform treatment decisions in the elderly, especially in the presence of comorbidities.

As might be expected, the number of lines of previous systemic therapy was a significant prognostic factor for PFS among pazopanib recipients in the PALETTE study (0-1 vs $2-4$ prior lines of therapy, HR [95\% CI]: 0.72 [0.53-0.99]; $P=0.04$ ) [2]. This finding is in agreement with "real-world evidence" demonstrating that patients with aSTS exhibit a decline in MPFS with each additional line of previous therapy [14]. The randomized, phase 2 EPAZ trial (NCT01861951) demonstrated that pazopanib was noninferior to doxorubicin with respect to PFS in the first-line treatment of STS in patients more than 60 years of age [15]. Recent studies have suggested that in some cases systemic anticancer therapy may promote progression of cancer rather than only influencing cancer evolution [16-18]. STS are characterized by tumor heterogeneity, and evolution of tumor heterogeneity in response to therapy is a well-established phenomenon [19]. Mutagenesis driven by cytotoxic therapies or by acquired chromosomal instability could drive clonal selection, leading to greater intratumoral heterogeneity and thereby increasing the likelihood of resistance to subsequent treatment [19]. Treatment sequence had no effect on pazopanib's safety profile, as evidenced by similar $\mathrm{AE}$ rates between the 2 subgroups based on prior lines of therapy.

In a subgroup analysis by pazopanib dose, patients receiving a daily dose between 400 and $600 \mathrm{mg}$ had higher mPFS than patients receiving a daily dose between 600 and $800 \mathrm{mg}$ or a daily dose of less than $400 \mathrm{mg}$; however, due to the small numbers of patients in the lower-dose subgroups, these results should be interpreted cautiously. Patients with metastatic renal cell carcinoma (RCC) receiving a lower starting dose of first-line

Table 3 Adverse Events in Pazopanib Recipients by Number of Prior Lines of Therapy (Safety Population)

\begin{tabular}{|c|c|c|}
\hline Events, n (\%) & $\begin{array}{l}1 \text { prior line } \\
(n=109)\end{array}$ & $\begin{array}{l}2+\text { prior lines } \\
(n=131)\end{array}$ \\
\hline Any on-therapy AE & $108(>99)$ & $129(98)$ \\
\hline AEs related to study treatment & $101(93)$ & $118(90)$ \\
\hline AEs leading to permanent discontinuation or early withdrawal & $24(22)$ & $24(18)$ \\
\hline AEs leading to dose reduction & $38(35)$ & $39(30)$ \\
\hline AEs leading to dose interruption/delay & $59(54)$ & $61(47)$ \\
\hline Any SAE & $47(43)$ & $52(40)$ \\
\hline SAEs related to study treatment & $26(24)$ & $31(24)$ \\
\hline Fatal SAEs & $5(5)$ & $3(2)$ \\
\hline Fatal SAEs related to study treatment & $0(0)$ & $1(<1)$ \\
\hline
\end{tabular}

$A E$ adverse event, $S A E$ serious adverse event. 
Table 4 Progression-Free Survival in Pazopanib Recipients by Dose Modifications (ITT Population)

\begin{tabular}{|c|c|c|}
\hline Dose Modifications & n (\%) & mPFS, weeks $(95 \% \mathrm{Cl})$ \\
\hline \multicolumn{3}{|l|}{ Dose reductions } \\
\hline No dose reduction & $154(100)$ & $11.9(8.9-19.3)$ \\
\hline Progressed or Died & $104(68)$ & \\
\hline$\geq 1$ dose reductions & $92(100)$ & $27.7(21.1-35.7)$ \\
\hline Progressed or Died & $59(64)$ & \\
\hline 1 dose reduction & $54(100)$ & $27.1(20.1-35.7)$ \\
\hline Progressed or Died & $29(54)$ & \\
\hline 2 or more dose reductions & $38(100)$ & $28.1(20.1-38.1)$ \\
\hline Progressed or Died & $30(79)$ & \\
\hline \multicolumn{3}{|l|}{ Dose interruptions } \\
\hline No dose interruption & $107(100)$ & $11.0(8.1-19.3)$ \\
\hline Progressed or Died & $71(66)$ & \\
\hline$\geq 1$ dose interruptions & $139(100)$ & $21.3(20.1-27.7)$ \\
\hline Progressed or Died & $92(66)$ & \\
\hline 1 dose interruption & $69(100)$ & $20.0(12.4-21.3)$ \\
\hline Progressed or Died & $45(65)$ & \\
\hline 2 or more dose interruptions & $70(100)$ & $27.7(21.0-35.6)$ \\
\hline Progressed or Died & $47(67)$ & \\
\hline
\end{tabular}

$\mathrm{Cl}$ confidence interval, ITT intention to treat

pazopanib fare worse compared with those receiving a standard dose [20]. At a median follow-up of 13.9 months, patients receiving reduced starting dose (400 or $600 \mathrm{mg} /$ day $)$ versus standard dose $(800 \mathrm{mg} /$ day $)$ have shown substantially reduced objective response rates (19\% vs $44 \%$, respectively) and increased discontinuation rates due to progressive disease $(44 \%$ vs $28 \%$, respectively) [20].

Intriguingly, patients receiving pazopanib with 1 or more dose reductions or dose interruptions because of drug toxicity had improved mPFS compared with patients in whom dose modifications were not required. These findings are consistent with those of the COMPARZ study in advanced renal cell carcinoma, wherein longer mPFS in pazopanib-treated patients was observed when dose modification was required because of toxicity, suggesting that patients are not at a disadvantage when such dose reductions or interruptions are needed [21]. In this context, "on-target" toxicities of TKIs have been suggested as potential indicators of efficacy [22]. In a pharmacokinetics/pharmacodynamics study in patients with RCC, the threshold concentration for pazopanib efficacy overlapped with concentrations at which toxicity occurs [23]. Evidence from previous reports and this study suggests that pazopanib recipients with no toxicity signs and symptoms (and thus not requiring a dose modification) may have suboptimal therapeutic drug exposure. However, the post hoc observational analysis in the current study did not account for the timing of dose modifications on treatment efficacy. A potential for bias due to early discontinuation in the groups with no dose reductions or interruptions subgroups cannot be ruled out, and hence, the effects of dose reductions and dose interruptions on mPFS outcomes need cautious interpretation.

This study has some additional limitations. Only data from subgroups of patients that received pazopanib were evaluated. All analyses were post hoc, descriptive, and exploratory in nature, and were not designed to permit statistical comparison across subgroups. For this reason, the possibility of bias in the descriptions of treatment effects in the post-randomization subgroups cannot be ruled out. Our findings therefore should be considered preliminary and will need to be confirmed in "realworld" settings.

\section{Conclusions}

In conclusion, longer mPFS was observed in patients receiving pazopanib as a second-line therapy for aSTS, rather than in later lines of treatment. Also, mPFS with pazopanib was maintained irrespective of patient age or the use of dose modifications for management of toxicity.

\section{Additional files}

Additional file 1: Table S1. Dose modification protocol for potential treatment-related adverse events in the PALETTE trial. This table describes the dose modification protocol used in the PALETTE trial for potential treatment-related adverse events. (DOCX 41 kb)

Additional file 2: Overview of study sites and their affiliated ethics committees. This table presents a list of all study sites/addresses and the affiliated ethics committees. (DOCX $32 \mathrm{~kb}$ )

\section{Abbreviations}

AE: Adverse event; aSTS: Advanced soft tissue sarcoma; Cl: Confidence interval; EMA: European Medicines Agency; GIST: Gastrointestinal stromal tumor; HR: Hazard ratio; IQR: Interquartile range; ITT: Intention to treat; mPFS: Median progression-free survival; OS: Overall survival; PDGF: Plateletderived growth factor; PDGFR: Platelet-derived growth factor receptor; PFS: Progression-free survival; RCC: Renal cell carcinoma; SAE: Serious adverse event; SD: Standard deviation; STS: Soft tissue sarcoma; TKI: Tyrosine kinase inhibitor; US FDA: United States Food and Drug Administration;

VEGF: Vascular endothelial growth factor; VEGFR: Vascular endothelial growth factor receptor

\section{Acknowledgments}

Financial support for medical editorial assistance was provided by Novartis Pharmaceuticals. We thank Vinay Pasupuleti, MD, PhD, ProEd

Communications, Inc., for his medical editorial assistance with this manuscript. We thank Dr. Arthur Staddon of University of Pennsylvania for his support and helpful review of this manuscript.

\section{Authors' contributions}

ALC contributed to the study concept, data acquisition, quality control of data and algorithms, data analysis and interpretation, and manuscript preparation, editing, and review. SB contributed to the study concept, data acquisition, quality control of data and algorithms, data analysis and interpretation, and manuscript preparation, editing, and review. GDD contributed to the study concept, study design, data acquisition, quality 
control of data and algorithms, data analysis and interpretation, and manuscript preparation, editing, and review. GH contributed to the quality control of data and algorithms, data analysis and interpretation, statistical analysis, and manuscript preparation, editing, and review. LD contributed to the quality control of data and algorithms, data analysis and interpretation, and manuscript preparation, editing, and review. QA contributed to the quality control of data and algorithms, data analysis and interpretation, and manuscript preparation, editing, and review. J-YB contributed to the study concept, study design, data acquisition, quality control of data and algorithms, data analysis and interpretation, and manuscript preparation, editing, and review. IJ contributed to the study concept, data acquisition, quality control of data and algorithms, data analysis and interpretation, and manuscript preparation, editing, and review. PS contributed to the study concept, data acquisition, quality control of data and algorithms, data analysis and interpretation, and manuscript preparation, editing, and review. MA contributed to the study concept, data acquisition, quality control of data and algorithms, data analysis and interpretation, and manuscript preparation, editing, and review. PH contributed to the study concept, data acquisition, quality control of data and algorithms, data analysis and interpretation, and manuscript preparation, editing, and review. HG contributed to the study concept, data acquisition, quality control of data and algorithms, data analysis and interpretation, and manuscript preparation, editing, and review. All authors gave their final approval of the version to be published, revised the manuscript critically and should have participated sufficiently in the work to take public responsibility for appropriate portions of the content. All authors agreed to be accountable for all aspects of the work in ensuring that questions related to the accuracy or integrity of any part of the work are appropriately investigated and resolved.

\section{Funding}

The PALETTE study was sponsored by GlaxoSmithKline Pharmaceuticals. GlaxoSmithKline and the European Organization for Research and Treatment of Cancer jointly conducted the study, including design, data collection, data analysis, and data interpretation. Pazopanib is an asset of Novartis AG as of March 1, 2015. Study investigators of both the EORTC and Novartis AG were involved in writing the report and in the decision to submit for publication. Novartis employees (listed as authors) were involved in current PALETTE subgroup analysis, interpretation and writing of the report. Financial support for medical editorial assistance was provided by Novartis Pharmaceuticals.

\section{Availability of data and materials}

Novartis is committed to sharing with qualified external researchers access to patient-level data and supporting clinical documents from eligible studies. These requests are reviewed and approved by an independent review panel on the basis of scientific merit. All data provided are anonymized to respect the privacy of patients who have participated in the trial in line with applicable laws and regulations.

\section{Ethics approval and consent to participate}

PALETTE (EORTC 62072; NCT00753688) was a randomized, double-blind, placebo-controlled, phase 3 trial, conducted by the Soft Tissue and Bone Sarcoma Group of the European Organization for Research and Treatment of Cancer between October 2008 and November 2010. An additional file provides an overview of all study sites and details on affiliated ethics committees (see Additional file 2). As part of the original PALETTE study, all patients provided written informed consent and the trial was approved by the institutional review board of each participating institution. Because the subgroup analyses used existing data from the primary study, additional consent was not required. The study was conducted in accordance with the Declaration of Helsinki and Good Clinical Practice guidelines.

\section{Consent for publication}

Not applicable.

\section{Competing interests}

ALC received honoraria from Pfizer, Lilly, Amgen, Novartis, PharmaMar, and Bayer.

SB received grants/research support from Novartis, Incyte, and Blueprint Medicine; received honoraria or consultation fees from Novartis, Lilly, Pfizer, PharmaMar, Deciphera, Bayer, and Nanobiotix.
GDD received consulting fees from Novartis, Pfizer, EMD-Serono, Sanofi Oncology, Janssen Oncology, Ignyta, Loxo Oncology, Mirati Therapeutics, Epizyme, PharmaMar, Daiichi-Sankyo, WIRB Copernicus Group, Ziopharm, and Polaris Pharmaceuticals; received research support to Dana-Farber from Bayer, Novartis, Pfizer, Janssen Oncology, Ignyta, Loxo Oncology, AbbVie, Epizyme, and Adaptimmune; patent licensed to Novartis from Dana-Farber with royalty paid to Dana-Farber; member, Board of Directors, Blueprint Medicines and Merrimack Pharmaceuticals; member, Scientific Advisory Board with consulting fees and equity, Blueprint Medicines and Merrimack Pharmaceuticals; consultant, Scientific Advisory Board with consulting fees and equity, G1 Therapeutics, Caris Life Sciences, and Champions Oncology; consultant with equity, Bessor Pharmaceuticals.

GH, LD, and QA are employees of Novartis Pharmaceuticals Corporation. $J-Y B$ received research support and honoraria from Novartis and GSK. IJ received honoraria from GSK for giving lectures at industry sponsored symposia.

$\mathrm{PH}$ received grants from Novartis; honoraria and consultation fees from GSK, Lilly, Pfizer, AROG, and PharmaMar.

PS received institutional support from providing consulting or an advisory role for 6th Element Capital, Adaptimmune, Amcure, Blueprint Medicines, BMS, Deciphera, Eisai, Eli Lilly, Ellipses Pharma, Epizyme, Genzyme, Ipsen, Loxo Oncology, Medpace, Merck, Nektar, Piqur Therapeutics, and Plexxikon; received institutional support for speaker's bureau from Eisai, PharmaMar, and Eli Lilly; received institutional support (research funding) from Blueprint Medicines, Boehringer Ingelheim, Cobiores nv, Eisai, Eli Lilly, Exelixis, G1Therapeutics, Novartis, PharmaMar, and Plexxikon; received institutional support for travel, accommodation, and expenses from 6th Element Capital, Adaptimmune, Amcure, AstraZeneca, Bayer, Blueprint Medicines, BMS, Boehringer Ingelheim, Daiichi Sankyo, Eisai, Eli Lilly, Epizyme, Genzyme, GSK, Ipsen, Loxo Oncology, Medpace, Nektar, Novartis, PharmaMar, Philogen, Piqur Therapeutics, and Plexxikon.

$\mathrm{MA}$ and $\mathrm{HG}$ declare that they have no competing interests.

\section{Author details}

'Département d'Oncologie Médicale, Gustave Roussy, 114 Rue Edouard Vaillant, 94805 Villejuif Cedex, Villejuif, France. ${ }^{2}$ Sarcoma Center, West German Cancer Center, University of Duisburg-Essen, Hufelandstraße 55, 45147 Essen, Germany. ${ }^{3}$ Ludwig Center at Harvard, Harvard Medical School and Department of Medical Oncology, Dana-Farber Cancer Institute, 450 Brookline Avenue, Boston, MA 02215, USA. ${ }^{4}$ Novartis Oncology, One Health Plaza, East Hanover, NJ 07936, USA. ${ }^{5}$ Department of Medical Oncology, Leon Berard Center, 28, rue Laennec 269373 Lyon Cedex 08, Lyon, France. ${ }^{6}$ The Institute of Cancer Research, Royal Marsden NHS Foundation Trust, 123 Old Brompton Road, London SW7 3RP, UK. 7Department of General Medical Oncology, University Hospitals Leuven, Leuven Cancer Institute, Herestraat 49, B-3000 Leuven, Belgium. ${ }^{8}$ Department of Oncology, University of Torino and Candiolo Cancer Center FPO-IRCCS, 10060 Candiolo, (Torino), Italy. ${ }^{9}$ Division of Surgical Oncology \& Thoracic Surgery, Mannheim University Medical Center, Theodor Kutzer Ufer 1, D-68165 Mannheim, Germany. ${ }^{10}$ Department of Medical Oncology, Leiden University Medical Center, Albinusdreef 2, 2333 Leiden, ZA, Netherlands.

Received: 16 November 2018 Accepted: 29 July 2019

Published online: 13 August 2019

\section{References}

1. Sonpavde G, Hutson TE. Pazopanib: a novel multitargeted tyrosine kinase inhibitor. Curr Oncol Rep. 2007;9:115-9.

2. van der Graaf WT, Blay JY, Chawla SP, Kim DW, Bui-Nguyen B, Casali $P G$, et al. Pazopanib for metastatic soft-tissue sarcoma (PALETTE): a randomised, double-blind, placebo-controlled phase 3 trial. Lancet. 2012;379:1879-86

3. Votrient (pazopanib) [prescribing information] https://www.accessdata.fda. gov/drugsatfda_docs/label/2009/022465/bl.pdf. Accessed 6 Sept 2018.

4. Votrient [Summary of Product characteristics] http://www.ema.europa.eu/ docs/en_GB/document_library/EPAR__PProduct_Information/human/ 001141/WC500094272.pdf. Accessed 6 Sept 2018.

5. Nijhuis PH, Schaapveld M, Otter R, Molenaar WM, van der Graaf WT, Hoekstra HJ. Epidemiological aspects of soft tissue sarcomas (STS)-consequences for the design of clinical STS trials. Eur J Cancer. 1999:35: 1705-10. 
6. Biau DJ, Ferguson PC, Turcotte RE, Chung P, Isler MH, Riad S, et al. Adverse effect of older age on the recurrence of soft tissue sarcoma of the extremities and trunk. J Clin Oncol. 2011;29:4029-35.

7. Hoven-Gondrie ML, Bastiaannet E, Ho VK, van Leeuwen BL, Liefers GJ, Hoekstra HJ, et al. Worse survival in elderly patients with extremity softtissue sarcoma. Ann Surg Oncol. 2016;23:2577-85.

8. Lev D, Pollock RE. Managing elderly soft tissue sarcoma patients-should age drive treatment? Ann Surg Oncol. 2010;17:1725-6.

9. Garbay D, Maki RG, Blay JY, Isambert N, Piperno Neumann S, Blay C, et al. Advanced soft-tissue sarcoma in elderly patients: patterns of care and survival. Ann Oncol. 2013;24:1924-30.

10. Hutchins LF, Unger JM, Crowley JJ, Coltman CA Jr, Albain KS. Underrepresentation of patients 65 years of age or older in cancertreatment trials. N Engl J Med. 1999;341:2061-7.

11. Subbiah V, Hess KR, Khawaja MR, Wagner MJ, Tang C, Naing A, et al. Evaluation of novel targeted therapies in aggressive biology sarcoma patients after progression from US FDA approved therapies. Sci Rep. 2016;6:35448.

12. Cesne AL, Judson I, Maki R, Grosso F, Schuetze S, Mehren MV, et al. Trabectedin is a feasible treatment for soft tissue sarcoma patients regardless of patient age: a retrospective pooled analysis of five phase II trials. Br J Cancer. 2013;109:1717-24.

13. Duffaud F, Ray-Coquard I, Marchal F, Chaigneau L, Bouche O, Huynh TK, et al. Clinical experience with sunitinib (SU) in patients over age 65 with metastatic gastrointestinal stromal tumors (GIST): a retrospective study from the French sarcoma group (FSG). J Clin Oncol. 2013;31:10546.

14. Ray-Coquard I, Collard O, Ducimetiere F, Laramas M, Mercier F, Ladarre N, et al. Treatment patterns and survival in an exhaustive French cohort of pazopanib-eligible patients with metastatic soft tissue sarcoma (STS). BMC Cancer. 2017;17:111

15. Grünwald V, Kunitz A, Schuler MK, Schoffski P, Kopp HG, Bauer S, et al. Randomized comparison of pazopanib (PAZ) and doxorubicin (DOX) in the first line treatment of metastatic soft tissue sarcoma (STS) in elderly patients (pts): results of a phase II study (EPAZ). J Clin Oncol 2018;36:11506.

16. Bolli N, Avet-Loiseau H, Wedge DC, Van Loo P, Alexandrov LB, Martincorena I, et al. Heterogeneity of genomic evolution and mutational profiles in multiple myeloma. Nat Commun. 2014;5:2997.

17. Johnson BE, Mazor T, Hong C, Barnes M, Aihara K, McLean CY, et al. Mutational analysis reveals the origin and therapy-driven evolution of recurrent glioma. Science. 2014;343:189-93.

18. Qazi MA, Vora P, Venugopal C, Sidhu SS, Moffat J, Swanton C, et al. Intratumoral heterogeneity: pathways to treatment resistance and relapse in human glioblastoma. Ann Oncol. 2017;28:1448-56.

19. Burrell RA, Swanton C. Tumour heterogeneity and the evolution of polyclonal drug resistance. Mol Oncol. 2014;8:1095-111.

20. Grassi P, Verzoni E, Ratta R, Porcu L, Prisciandaro M, Mennitto A, et al. Does dose modification affect efficacy of first-line pazopanib in metastatic renal cell carcinoma? Drugs R D. 2017;17:461-7.

21. Bjarnason GA, Kollmannsberger CK, Ahmad Q, Dezzani L, Elmeliegy M, Han J, et al. Effects of pazopanib (PAZ) and sunitinib (SUN) dose modification on safety and efficacy in patients with metastatic renal cell carcinoma (mRCC) from COMPARZ. J Clin Oncol. 2017;35:4574.

22. Shah DR, Shah RR, Morganroth J. Tyrosine kinase inhibitors: their on-target toxicities as potential indicators of efficacy. Drug Saf. 2013;36:413-26.

23. Suttle AB, Ball HA, Molimard M, Hutson TE, Carpenter C, Rajagopalan D, et al. Relationships between pazopanib exposure and clinical safety and efficacy in patients with advanced renal cell carcinoma. Br J Cancer. 2014; 111:1909-16.

\section{Publisher's Note}

Springer Nature remains neutral with regard to jurisdictional claims in published maps and institutional affiliations.

Ready to submit your research? Choose BMC and benefit from:

- fast, convenient online submission

- thorough peer review by experienced researchers in your field

- rapid publication on acceptance

- support for research data, including large and complex data types

- gold Open Access which fosters wider collaboration and increased citations

- maximum visibility for your research: over $100 \mathrm{M}$ website views per year

At BMC, research is always in progress.

Learn more biomedcentral.com/submissions 